\title{
Impedance Spectroscopy of Supported Multiwalled Carbon Nanotubes for Immunosensor Applications
}

\author{
Imen Hafaiedh ${ }^{1 *}$, Sami Ameur ${ }^{2}$, Adnane Abdelghani ${ }^{1}$
}

${ }^{1}$ Carthage University, Nanotechnology Laboratory, National Institute of Applied Science and Technology, centre Urbain Nord, Bp676, 1080 Charguia Cedex, Tunisia ${ }^{2}$ University of Sousse, Higher Institute of Agronomic Sciences of Chatt-Meriem, BP 47 ; 4042 Chatt-Meriem, Tunisia

\begin{abstract}
In this work, we used carboxyl multiwall carbons nanotubes for immunosensor application. The carbon nanotubes functionalisation can be achieved with Pressure Vapor Deposition (PVD) technique. The obtained carbon nanotubes can be immobilized on gold electrode with the airbrushing technique. The morphology of the deposited carboxyl carbon nanotubes was observed with Scanning Electron Microscopy (SEM) and Transmission Electron Microscopy (TEM). The electrochemical characterization of the carboxyl multiwall carbon nanotubes was achieved with cyclic voltammetry and impedance spectroscopy. It shows a higher resistance and a good stability in water interface. For anti-rabbit IgG immobilization, the carboxyl carbon nanotubes were activated with EDC/NHS and non-specific sites was blocked with Bovine Serum Albumin (BSA). The development of immunosensor for lgG detection was observed with the impedance spectroscopy. The results show that the immunosensor response depends on the conductivity and the large surface-tovolume ratio attained with multiwall carbon nanotubes. The response of the developed immunosensor was reproducible, with higher stability and with limit detection of $10 \mathrm{pg} / \mathrm{ml}$ antigen.
\end{abstract}

Keywords: Carboxyl multiwalled carbon nanotubes; Immunosenors; Impedance spectroscopy; Cyclic voltammetry

\section{Introduction}

Owing to their nano-dimensions, rich electronic states, large surface area, high mechanical strength, and excellent chemical and thermal stability, carbon nanotubes have attracted a great deal of interest [1]. Among the many potential applications [1-3], carbon nanotubes have recently become promising functional materials for the development of advanced biosensors [2] and gas sensors [4-6] with novel features.

There are multiple possibilities for surface modification [7] of carbon nanotubes, but functionalization with antibodies has gained significant importance in recent years. Antibodies not only provide the required biocompatibility [8] but also alter the electrical and optical properties of nanotubes and make their surface sensitive to surroundings $[8,9]$.

In previous work [10-12], we used carboxyl modified thiol monolayers for immunosensor applications. We used impedance spectroscopy and quartz microbalance as transduction techniques. The sample preparation was time consuming and we obtained a higher limit detection [10-12].

In this work, we used carboxyl multiwall carbon nanotubes for immunosensor application. The obtained carbon nanotubes can be immobilized on gold electrode with the airbrushing technique. The morphology of the deposited carboxyl carbon nanotubes was observed with Scanning Electron Microscopy (SEM) and Transmission Electron Microscopy (TEM). The electrochemical characterization of the carboxyl multiwall carbon nanotubes were achieved with cyclic voltammetry and impedance spectroscopy. The results show that the immunosenosr response depends on the conductivity and the large surface-to-volume ratio attained with multiwall carbon nanotubes. The response of the developed immunosensor was reproducible, with higher stability and with limit detection of $10 \mathrm{pg} / \mathrm{ml}$ antigen.

\section{Materials and Methods}

\section{Reagents}

1-ethyl-3-(3-(dimethylamino)-propyl)carbodiimide (EDC), N-hy- droxy succinimide (NHS) (Aldrich) and Albumin from Bovine Serum (BSA) were obtained from sigma Aldrich. The Goat anti-rabbit IgG and Rabbit IgG were purchased from Pierce Biotechnology. The MWCNTs (Nanocyl, Belgium) were grown by PVD (Pressure Vapor Deposition) with purity higher than $95 \%$. Nanotubes were upto 50 micrometer in length and their outer and inner diameters ranged from $3 \mathrm{~nm}$ to $15 \mathrm{~nm}$ and $3 \mathrm{~nm}$ to $7 \mathrm{~nm}$, respectively.

The buffer solution used for all experiments was phosphate buffered saline (PBS) containing $140 \mathrm{mM} \mathrm{NaCl}, 2.7 \mathrm{mM} \mathrm{KCl}, 0.1 \mathrm{mM} \mathrm{Na}_{2} \mathrm{HPO}_{4}$ $1.8 \mathrm{mM} \mathrm{KH}_{2} \mathrm{PO}_{4}, \mathrm{pH}=7$ and the redox couple $\mathrm{Fe}(\mathrm{CN})_{6}^{-3} / \mathrm{Fe}(\mathrm{CN})_{6}^{-4}$ at a $5 \mathrm{mM}$ concentration. All reagents were of analytical grade and ultrapure water (resistance $18.2 \mathrm{M} \Omega \mathrm{cm}^{-1}$ ) produced by a MilliporeMilli-Q system was used.

\section{Carboxyl MWCNT synthesis}

The MWCNT used in the experiment were obtained from Nanocyl, S.A. They were synthesized by chemical vapor deposition and their purity was higher than $95 \%$. The nanotubes were upto 50 microns in length and their outer and inner diameters ranged from 3 to $15 \mathrm{~nm}$ and 3 to $7 \mathrm{~nm}$, respectively.

A uniform functionalization with carboxylic group was applied to the as provided carbon nanotubes in order to improve their dispersion and surface reactivity. More details can be found in reference [2]. In the second processing step, the functionalized carbon nanotubes were

*Corresponding author: Imene Hafaiedh, Nanotechnology Laboratory, National Institute of Applied Science and Technology centre, Urbain Nord, Bp676, 1080 Charguia Cedex, Tunisia, Tel: +216 703 829; Fax: +216 70704 329; E-mail: imen_haf@yahoo.fr

Received September 26, 2012; Accepted October 16, 2012; Published October 20, 2012

Citation: Hafaiedh I, Abdelghani A (2012) Impedance Spectroscopy of Supported Multiwalled Carbon Nanotubes for Immunosensor Applications. J Nanomedic Nanotechnol 6:275. doi:10.4172/2157-7439.1000275

Copyright: (c) 2012 Hafaiedh I, et al. This is an open-access article distributed under the terms of the Creative Commons Attribution License, which permits unrestricted use, distribution, and reproduction in any medium, provided the original author and source are credited. 
Citation: Hafaiedh I, Abdelghani A (2012) Impedance Spectroscopy of Supported Multiwalled Carbon Nanotubes for Immunosensor Applications. J Nanomedic Nanotechnol 6:275. doi:10.4172/2157-7439.1000275

dispersed in an organic vehicle (dimethylformamide), ultrasonically stirred during $20 \mathrm{~min}$ at room temperature and subsequently airbrushed onto alumina sensor substrates while controlling the resistance of the resulting film during the deposition. These substrates consist of gold electrode. The active films coated the membrane areas through a shadow mask. During deposition, substrates were kept at $100^{\circ} \mathrm{C}$, which ensured a fast evaporation of the solvent.

\section{Gold Cleaning and Functionalization}

The gold electrodes $(1 \mathrm{~cm} \times 1 \mathrm{~cm})$ were fabricated at the National Center of Microelectronics of Barcelona (Spain). Evaporated gold ( 300 $\mathrm{nm}$ thickness) was deposited on silicon, using a titanium underlayer ( $\sim 30 \mathrm{~nm}$ thickness) as substrate. Before modification, the gold electrodes were cleaned in acetone solution for $10 \mathrm{~min}$ with ultrasonic bath. After that, they were dried under a nitrogen flow and then dipped for 10 min into "piranha solution" $7: 3$ (v/v) $96 \% \mathrm{H}_{2} \mathrm{SO}_{4} / 30 \% \mathrm{H}_{2} \mathrm{O}_{2}$. Finally, the gold substrates were rinsed 2 to 3 times with ultrapure water and immediately immersed in an ethanol solution. After cleaning, the functionalized multiwall carbon nanotubes were deposited on gold electrode with the Airbrushing technique at $90^{\circ} \mathrm{C}$ [5]. Immediately the substrate was treated for two hours at $120^{\circ} \mathrm{C}$. More details concerning the synthesis and deposition of the gold labeled multiwall carbon nanotubes could be found [6].

After this, the modified gold electrode was immersed in a solution of EDC $(0.4 \mathrm{mM})$ and NHS $(0.1 \mathrm{mM})$ for $1 \mathrm{~h}$. A $500 \mu \mathrm{l}$ of Goat antirabbit IgG solution with a concentration of $5 \mu \mathrm{g} / \mathrm{ml}$ was deposited on the treated electrode for 1 hour. Then, a $500 \mu \mathrm{l}$ of $1 \%$ BSA solution was added on the substrate for 30 min to block the free spaces between the antibody and multiwall carbon nanotubes [10-13]. Antigen at different concentrations was incubated on immobilized antibody for 30 minutes at room temperature [11-13]. Figure 1 shows the schematic representation of the fabrication process of the multilayer structure of the immunosensor.

\section{Electrochemical Set-Up}

Cyclic Voltammetry: Cyclic voltammetry was performed at room temperature in a conventional voltammetric cell with a three electrode configuration using Autolab impedance anlayzer (Ecochemie, Netherland). The gold electrode $\left(0.16 \mathrm{~cm}^{2}\right)$ was used as working electrode, platinum $\left(1 \mathrm{~cm}^{2}\right)$ and $\mathrm{Ag} / \mathrm{Ag}$ - $\mathrm{Cl}$ electrodes were used as counter and reference electrodes respectively. All the electrochemical measurements were carried out in PBS at $\mathrm{pH} 7.0$ with $5 \mathrm{mM}$ $\mathrm{Fe}(\mathrm{CN})_{6}^{-3} / \mathrm{Fe}(\mathrm{CN})_{6}^{-4}$ and in Faraday cage [13-20].

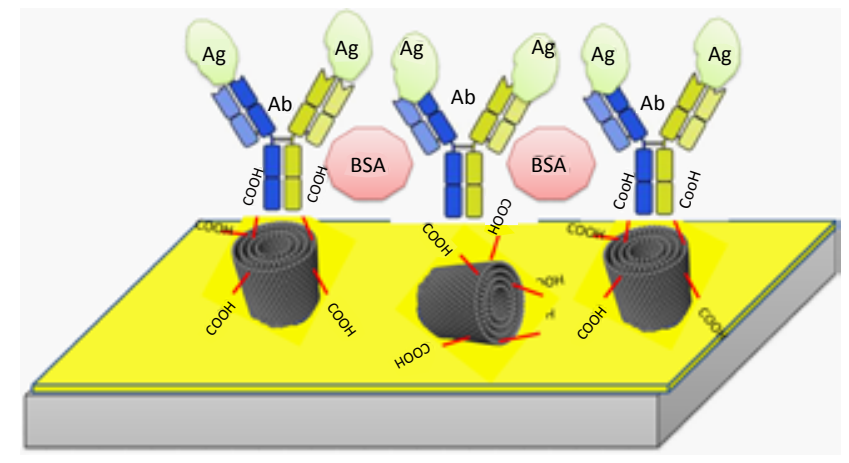

Figure 1: Schematic representation of the fabrication process of the multilayer system of the immunosensor on the gold electrode.
Impedance Spectroscopy: In a number of reports [12-20], it has been shown that impedance spectroscopy is a useful tool to characterise the compactness amphiphilic films on solid state surfaces. A capacitor is formed between the conducting electrode and the electrolyte. The frequency dependent capacitance $\mathrm{C}$ of the complex stratified surface is related to the absolute value of the complex electric impedance $\mathrm{Z}$ (measured in Ohms) by the equation:

$$
|Z|=\frac{1}{2 \pi f C}
$$

Where $\mathrm{f}$ is the frequency (in $\mathrm{Hz}$ ) at which $|Z|$ is measured.

The surface of the device with a supported film in contact with the aqueous phase exhibits a complex capacitance composed of atleast three sub-layers. If the complex impedance $Z_{i}$ of the sub-layers $i$ can be determined by analysis of the measured impedance in terms of an electrical equivalent circuit, the thickness of the sub-layers (i) can be determined according to equation:

$$
d_{i}=\frac{\mathcal{E}_{0} \mathcal{E}_{i} \mathrm{~A}}{\mathcal{C}_{i}}
$$

$\varepsilon 0$ is the vaccum dielectric constant $\left(8.85 \times 10^{-14} \mathrm{~F} / \mathrm{cm}^{2}\right), \varepsilon I$ is the dielectric constant of the layer $i$ and $A$ is the area of the surface. To fit the measured spectra with the impedance spectra out of ideal elements, we replaced the ideal elements with the constant phase elements (CPE) :

$$
Z_{C P E}=K \omega^{-a}
$$

The frequency exponent is $\alpha=1$ and $\mathrm{K}=1 / \mathrm{C}$ for an ideal capacitance, and $\alpha=0$ and $K=R$ for an ideal resistance, respectively. The exponent $\alpha$ could be obtained, when the membrane capacitance (or layer capacitance) was replaced by a constant phase element $Z_{C P E}$. The deviation of the exponent $\alpha$ from the ideal values is attributed to the inhomogeneities of the analyzed layer, like defects or roughness. The measured spectra of the impedance and phase were analyzed in terms of electrical equivalent circuits using an $Z_{\text {view }}$ software. The electric parameters of the system were calculated with the computer program and the fit error was kept under a maximum of $10 \%$.

The impedance measurement was performed in the frequency range of $0.05 \mathrm{~Hz}$ to $100 \mathrm{kHz}$, using a modulation voltage of $10 \mathrm{mV}$. More details on electrochemical impedance spectroscopy can be found in reference [15-20].

\section{Results and Discussion}

\section{Characterization of multiwall carbon nanotubes layer}

Morphological characterization of CNT films: The morphology of the functionalized MWCNT films was investigated with scanning electron microscopy and transmission electron microscopy (Figure 2). The MWCNT nanopores of two-dimensional arrays with different size and with homogenous distribution were observed.

Cyclic voltammetry: Cyclic voltammetry is an electrochemical technique which can be used to study the kinetic of redox reactions of materials, their insulating and conducting properties. Cyclic voltammograms of the gold electrode figure $3 a$, shows a reversible wave which is the typical behavior of gold surface in the presence of the redox couple. As is shown in (Figure 3a), the cyclic voltammogram defines the characteristics of a diffusion controlled redox process observed at the cleaned bare gold electrode. After modification of the gold 
Citation: Hafaiedh I, Abdelghani A (2012) Impedance Spectroscopy of Supported Multiwalled Carbon Nanotubes for Immunosensor Applications. J Nanomedic Nanotechnol 6:275. doi:10.4172/2157-7439.1000275

Page 3 of 5

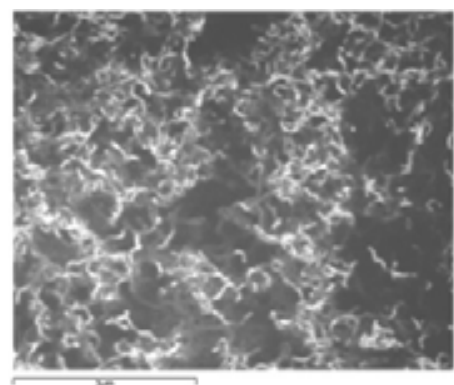

(a)

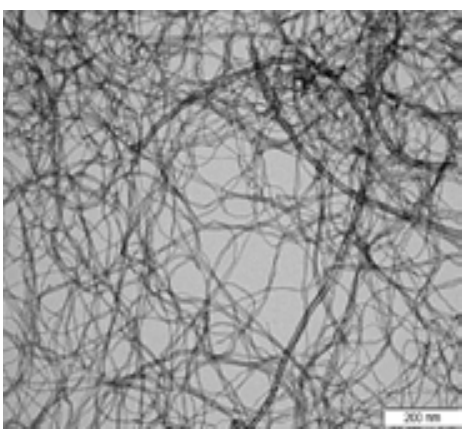

(b)

Figure 2: (a) ESCM image (b) TEM image.

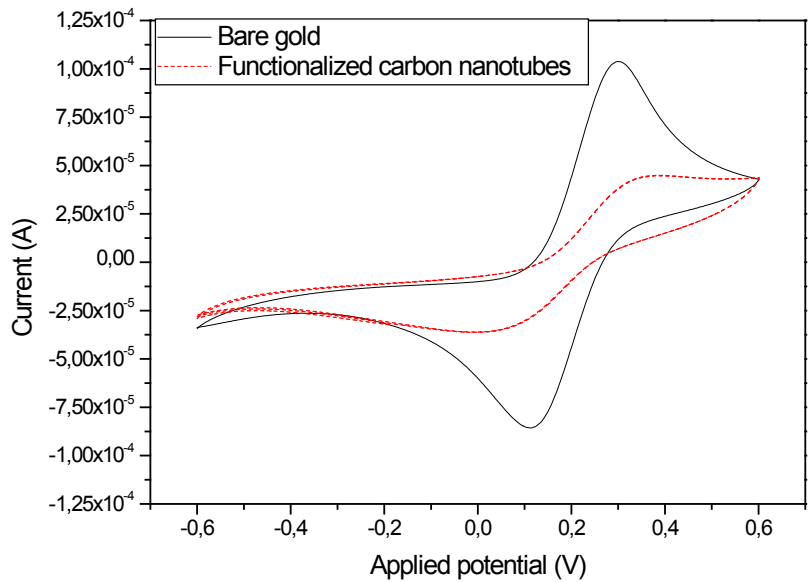

Figure 3: (a): Cyclic voltammograms of the gold electrode shows a reversible wave which is the typical behavior of gold surface in the presence of the redox couple; (b): Cyclic voltammograms of the gold electrode after MWCNTs deposition shows an decrease of the current.

surface with the functionalized multiwall carbon nanotube layer, the current decrease due to the insulating properties of the functionalized MWCNTs layer (Figure 3b). The success immobilization of multiwall carbon nanotube layer was confirmed with the current decreases.

Impedance Measurement: Electrochemical Impedance Spectroscopy (EIS) is an effective tool for probing the feature surface-modified electrode while controlling its electrical properties [15-20]. This technique was used for example for the characterization of impedance behavior of SAM modified gold electrode [15-18]. Figure 4 shows Nyquist plots for gold electrode before and after MWCNTs deposition at $200 \mathrm{mV}$ with redox couple, where $\operatorname{Re}(z)$ is the real part and $\operatorname{Im}(z)$ is the imaginary part of the complex impedance $Z$. The diameter of semi- circle corresponds to the charge transfer resistance of the electrode/ electrolyte interface. The impedance spectra should be fitted using the electric model presented in figure 5 (Randle's equivalent circuit). The $\mathrm{CPE}$ is the Constant Phase Element impedance of the gold/electrolyte interface, $R_{m}$ is the charge transfer resistance in low frequency range and $\mathrm{W}$ is the impedance due to the mass transfer of the redox species to the electrode described by Warburg (Table 1). The resistance in high frequency ranges $\mathrm{R}_{\mathrm{s}}$ is the resistance of the electrolyte, the contacts and connections.

The increase of the charge transfer resistance after MWCNTs immobilization (Figure $4 \mathrm{~b}$ ) is due to the higher conductivity decreases at the gold-electrolyte interface. This confirms the results obtained with cyclic voltammetry. The charge transfer resistance before and after MCWNTs deposition were extracted from the fitted data (Table 1).

\section{Anti-IgG immobilization on multiwall carbon Nanotubes}

Cyclic voltammetry: Figure 6 shows the cyclic voltammograms of the gold electrode with MWCNTs (Figure 5a), with immobilized antiIgG (Figure 6b) and with BSA (Figure 6c). The immobilization of anti-

\begin{tabular}{|l|l|l|l|l|}
\hline & $\mathrm{R}_{\mathrm{s}}\left(\Omega \cdot \mathrm{Cm}^{2}\right)$ & $\mathrm{R}_{\mathrm{m}}\left(\Omega \cdot \mathrm{Cm}^{2}\right)$ & $\mathrm{CPE}\left(\mathrm{F} . \mathrm{cm}^{2}\right)$ & $\mathrm{W}\left(\Omega . \mathrm{Cm}^{2}\right)$ \\
\hline Gold & 259,456 & 134,980 & $2,197.10^{-5}$ & 1645,035 \\
\hline Gold+ MWCNTs & 259,124 & 7185,340 & $5,132.10^{-5}$ & 293,132 \\
\hline $\begin{array}{l}\text { Gold+ MWCNTs+ anti-lgG } \\
\begin{array}{l}\text { Gold+ MWCNTs + anti- } \\
\text { IgG+BSA }\end{array}\end{array}$ & 261,034 & 221,654 & $1,034.10^{-5}$ & 1734,181 \\
\hline $\begin{array}{l}\text { Gold+MWCNTs+anti- } \\
\text { IgG+BSA+ Ag (10pg/ml })\end{array}$ & 260,305 & 590,045 & $1,980.10^{-5}$ & 1801,445 \\
\hline
\end{tabular}

Table1: The electrical parameters of Randle's circuit obtained with the fitting program. The fit error was kept under a maximum of $10 \%$.

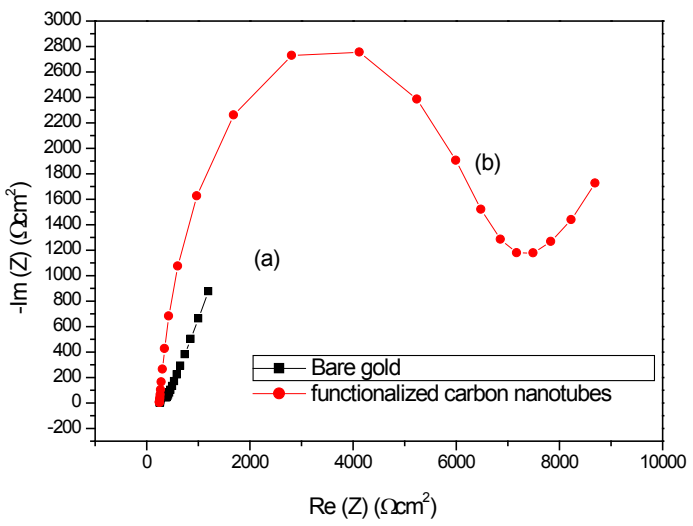

Figure 4: (a): Nyquist impedance plots for gold electrode with redox couple at $200 \mathrm{~V}$; (b): Nyquist impedance plots for gold electrode after MWCNTs deposition with redox couple at $200 \mathrm{~m} \mathrm{V.}$

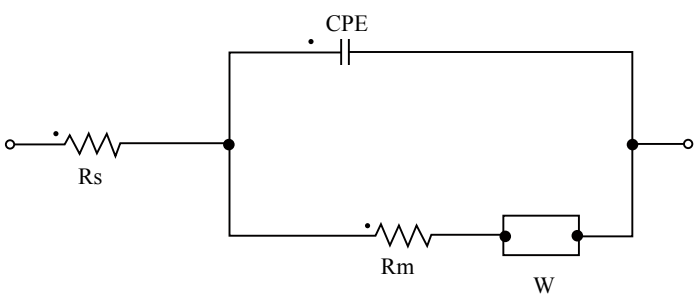

Figure 5: Electric model. 
Citation: Hafaiedh I, Abdelghani A (2012) Impedance Spectroscopy of Supported Multiwalled Carbon Nanotubes for Immunosensor Applications. J Nanomedic Nanotechnol 6:275. doi:10.4172/2157-7439.1000275

Page 4 of 5

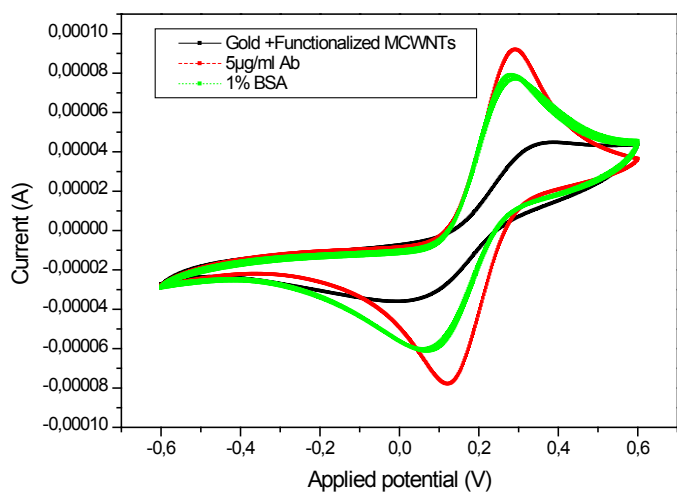

Figure 6: (a): Cyclic voltammograms of the gold electrode with MWCNTs; (b): Cyclic voltammograms of the gold elec- trode with MWCNTs after Antibody deposition; (c): Cyclic voltammograms of the gold electrode with MWCNTs $\mathrm{Ab}$ and $\mathrm{BSA}$.

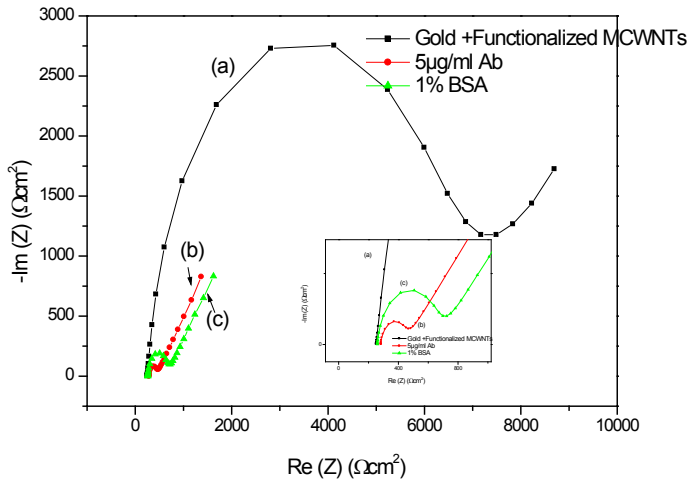

Figure 7: (a): Nyquist impedance plots for gold electrode with MWCNTs at $200 \mathrm{mV}$; (b): Nyquist impedance plots for gold electrode with MWCNTs after Antibody immobilization at $200 \mathrm{mV}$; (c): Nyquist impedance plots for gold electrode with MWCNTs BSA immobilization at $200 \mathrm{mV}$.

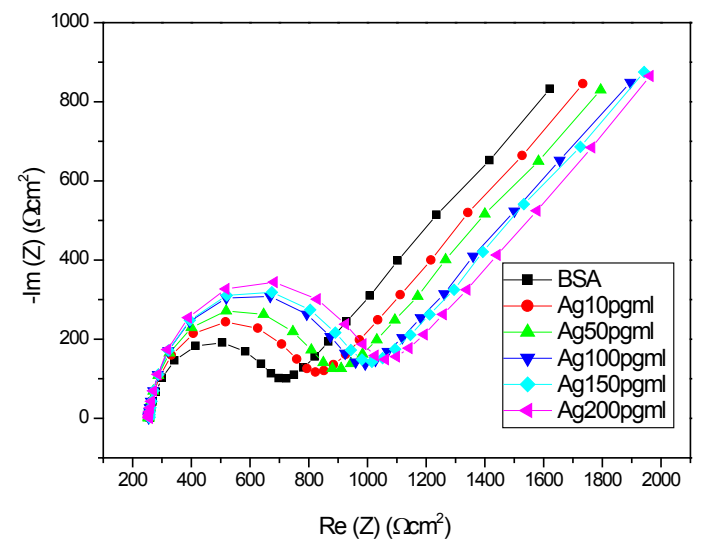

Figure 8: Nyquist impedance plots for gold electrode with MWCNTs after antigen injections.

IgG induce an increase in the current due to the iso-electric point of the used antibody. The deposition of BSA blocking layer induce a decrease in the current.

Impedance spectroscopy: Figure 7 shows the Nyquist plot of the gold electrode with MWCNTs (Figure 7a), with immobilized anti-IgG (Figure 7b) and with BSA (Figure 7c). The immobilization of antiIgG induce a decrease in the charge transfer resistance with confirm the cyclic voltammetry results. The deposition of BSA blocking layer induces an increase in the charge transfer resistance which confirm the cyclic voltammetry results.

The Constant phase element impedance, the charge transfer resistance in low frequency range and the warburg impedance for each deposition steps was shown in table 1.

Immunosensor application: Figure 8 shows the impedance spectra of the gold electrode functionalized with anti-IgG (with BSA as blocking layer) before and after injection of IgG concentrations. The semicircle diameter in the Nyquist plot seems to increase with the antigen concentration, implying that more amount of antigen was linked to the interface. When the concentration of antigen was increased over to $100 \mathrm{pg} / \mathrm{ml}$, impedance spectra changes become gradually slow, showing that the immobilization of antigen on gold electrode trends to be saturated.

In order to obtain the calibration data set, the values of electrode resistance variation $\Delta \mathrm{R}_{\mathrm{m}}$ versus the antigen concentration were plotted in figure 9 (curve a). The change of membrane resistance is calculated following the equation:

$$
\Delta \mathrm{R}_{\mathrm{m}}=\mathrm{R}_{\mathrm{m}(\mathrm{Ab})}-\mathrm{R}_{\mathrm{m}(\mathrm{Ab}-\mathrm{Ag})}
$$

Where $R_{m(A b)}$ is the value of electrode resistance as antibody immobilized on the electrode, $R_{m(A b-A g)}$ is the value of the electrode resistance after antigen binding to antibody. As we can see in the figure 9a, the plot is linear and presents saturation for higher IgG concentration. A detection limit of $10 \mathrm{pg} / \mathrm{ml}$ antigen was obtained. These results show that the immunosensor response depends on the conductivity and the large surface-to-volume ratio attained with multiwall carbon nanotubes $[21,22]$.

To confirm that the above observed impedance changes generated from the result of specific antibody-antigen interaction, the sensor was exposed to the solution of sheep IgG that are expected to bind nonspecifically. Figure 9 (curve b) shows the variation of the electrode resistance versus the non-specific antigen concentration. As we can see,

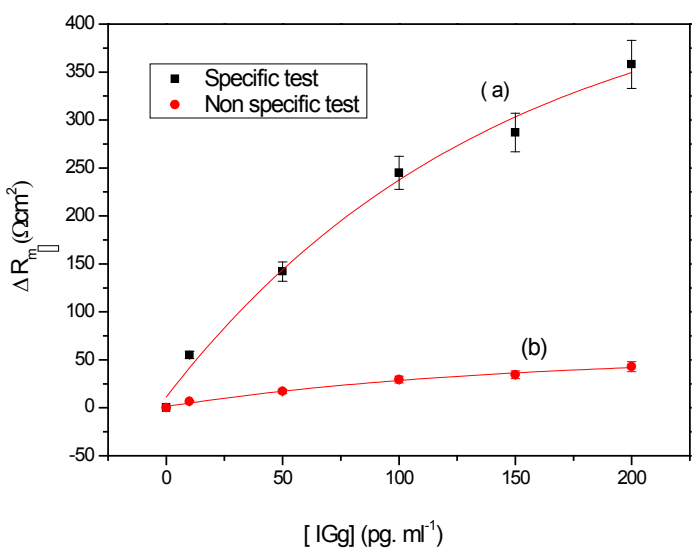

Figure 9: Calibration curve for specific and non-specific detection. 
Citation: Hafaiedh I, Abdelghani A (2012) Impedance Spectroscopy of Supported Multiwalled Carbon Nanotubes for Immunosensor Applications. J Nanomedic Nanotechnol 6:275. doi:10.4172/2157-7439.1000275

the sensor was not subjected to the non-specific binding and applicable to the selective determination of IgG.

\section{Conclusion}

In this work, we used gold labeled functionalized multiwall carbon nanotubes for immunosensors applications. The labeled carboxyl carbon nanotubes can be immobilized on gold electrode with the Airbrushing technique. The stability and the molecular structure of the labeled multiwall carbon nanotubes were characterized with cyclic voltammetry, impedance spec-troscopy, Scanning Electron Microscopy (SEM) and Transmission Electron Microscopy (TEM). For Anti-IGg immobilization, the labeled carboxyl carbon nanotubes were activated with EDC/NHS. The developed biosensor can detect IGg concentration in the range $10 \mathrm{pg} \mathrm{ml}^{-1}-100 \mathrm{pg} \mathrm{ml}^{-1}$. The results show that the Immunosensor response depends on the conductivity and the large surface-to-volume ratio attained with multiwall carbon nanotubes. The response of the developed Immunosensor was reproducible and with higher stability.

\section{References}

1. Rao CN, Satishkumar BC, Govindaraj A, Nath M (2001) Nanotube. Chem Phys Chem 79-105.

2. Wang J (2005) Carbon-Nanotube Based Electrochemical Biosensors: A Review. Electroanalysis 17: 7-14.

3. Baughman RH, Zakhidov A, Heer WA (2002) Carbon Nanotubes: the Route Toward Applications. Science 297: 787-792.

4. Star A, Joshi V, Skarupo S, Thomas D (2006) Gas sensor array based on metal-decorated carbon nanotubes, Journal Physical Chemistry B 110: 21014 21020

5. Jimeneza I, Cirera A, Cornet A, Morante JR, Garcia I, et al. (2002) Pulverisation method for active layer coating on microsystems. Sensors and Actuators B 84 : 78-82.

6. Radhouane Legrib (2010) Design, Fabrication and Characterization of Gas Sensors Based on Nanohybrid Materials, Ph.D Dissertation, Tarragona (Spain), ISBN: 978-84-694-0326-6.

7. Ramanathan K, Bangar MA, Yun M, Chen W, Myung NV, et al. (2005) Bioaffinity sensing using biologically functionalized conducting-polymer nanowire. J Am Chem Soc 127: 496-497.

8. Elkin T, Jiang X, Taylor S, Lin Y, Gu L, et al. (2005) Immuno-carbon nanotubes and recognition of pathogens. Chem Bio Chem 6: 640-643.
9. Xu Z, Hu P, Wang S, Wang X (2008) Biological functionalization and fluorescent imaging of carbon nanotubes. Appl Surf Sci 254: 1915-1918.

10. Tlili A, Abdelghani A, Hleli S, Maaref MH (2004) Electrical Characterization of a Thiol SAM on Gold as a First Step of the Fabrication of an Immunosensors based on a Quartz Crystal Microbalance. Sensors 4: 105-114.

11. Tlili A, Abdelghani A, Jarboui MA, Dahmani F (2005) A Novel Silicon Nitride Biosensor for Specific Antibody-Antigen interaction. Materials Science and Engineering C 25: 490-495.

12. Tlili A, Abdelghani A, Ameur S, Renault NJ (2006) Impedance Spectroscopy and Affinity Measurement of Specific Antibody-Antigen Interaction. Mater Sc Eng C 26: 546-550.

13. Abdelghani A, Hleli S, Cherif K (2002) Optical and electrochemica characterization of self assembled octadecyltrichlorosilane monolayer on modified silicon electrode. Material letters 56: 1064-1068.

14. Hou Y, Helali S, Zhang A, Jaffrezic-Renault N, Martelet C, et al. (2006) Immobilization of Rhodopsin on a self-assembled multilayer and its specific detection by electrochemical impedance spectroscopy. Biosensors and Bioelectronics 21: 1393-1402.

15. Hleli S, Abdelghani A, Tlili A (2003) Impedance Spectroscopy Technique for DNA Hybridization. Sensors 3: 472-479.

16. Hleli S, Martelet C, Abdelghani A, Burais N, Renault NJ (2006) Atrazine analysis using an impedimetric immunosensor based on mixed biotinylated self-assembled monolayer. Sensors and Actuators B 113: 711-717.

17. MacDonald JR (1990) Impedance Spectroscopy-old problems and new developments. Wiley, New York.

18. Hillebrandt H, Abdelghani A, Abdelghani C, Sackmann E (2001) Electrical and optical characterization of thrombin-induced permeability of cultured endothelia cell monolayers on semiconductor electrode arrays. Applied Physics A 73: 539546.

19. Wiegand Gerlald (1999) Fundamental principles of the electric properties of supported lipid membranes investigated by advanced methods of impedance spectroscopy, Ph.D dissertation, Shaker verlag, ISBN 3-8265-7231-9, Technishe Universität of München, Germany.

20. Hafaid I, Chebil S, Youssoufi HK, Bessueille F, Errachid A, et al. (2010) Effect of electrical conditions on an impedimetric immunosensor based on a modified conducting polypyrrole. Sens Actuators B Chem 144: 323-331.

21. B.Vigolo, A.Pénicaud, C. Coulon, C. Sauder, R. Pailler, C. Journet, P. Bernier , P. Poulin Macroscopic fibers and ribbons of oriented carbon nanotubes Science. 2000, 290, pp.1331-1334

22. G. Mago,F. T. Fisher,D. M. Kalyon, Effects of Multiwalled Carbon Nanotubes on the Shear-Induced Crystallization Behavior of Poly(butylene terephthalate) Macromolecules 2008, 41, pp. 8103-8113.
This article was originally published in a special issue, Functionalized nanomaterials: Biomedical and Sensing Applications handled by Editor(s). Dr. Sadeghi SM, University of Alabama in Huntsville, USA 